\title{
SINGULAR RULED SURFACES IN SPACE OF FIVE DIMENSIONS*
}

\author{
BY \\ E. B. STOUFFER
}

In a previous paper $\dagger$ the author has made a study of the projective differential properties of ruled surfaces in space of five dimensions by means of a system of two linear homogeneous differential equations of the third order. The method gave results only for regular, or non-singular, ruled surfaces, that is, for surfaces which do not have in general three consecutive generators in the same 4-space. However, the singular surfaces are of even greater interest than the regular surfaces. This is particularly true because surfaces in 4-space and even in 3-space may be regarded as such singular surfaces and, consequently, generalizations of some of the properties of ruled surfaces in 3-space become quite apparent. In this paper a plan has been devised for studying all ruled surfaces in 5 -space except those which have in general consecutive generators intersecting, that is, which are developable in the ordinary sense. Since the regular surfaces in 5-space have already been treated in the paper mentioned, the study in this paper will be confined largely to non-developable singular ruled surfaces, including surfaces in 4-space but not in 3-space.

The method here used may be immediately generalized to provide for the study of any spread generated by $\infty^{1}$ linear spaces in space of any number of dimensions, provided merely that two consecutive generating spaces do not in general have a point in common. Moreover, the author in previous papers $\ddagger$ has set up much of the necessary analytical machinery for such studies.

* Presented to the Society, December 27, 1918, and November 26, 1921; received by the editors in April, 1926.

$\dagger$ Invariants of linear homogeneous differential equations, with applications to ruled surfaces in five-dimensional space, Proceedings of the London Mathematical Society, (2), vol. 11 (1913), pp. 185-224.

$\ddagger$ On seminvariants of linear homogeneous differential equations, Proceedings of the London Mathematical Society, (2), vol. 15 (1916), pp. 217-226; On invariants and covariants of linear homogeneous differential equations, Proceedings of the London Mathematical Society, (2), vol. 17 (1919), pp. 337-352. This second paper will be referred to in the future as Invariants and covariants. 
The papers most closely related to the present work are three by Ranum* published from 1912 to 1915, and one by Bompiani† published in 1914.

\section{THE EQUATIONS AND THEIR TRANSFORMATIONS}

The system of linear homogeneous differential equations

$$
y_{i}^{\prime \prime}+2 \sum_{k=1}^{3} p_{i k} y_{k}{ }^{\prime}+\sum_{k=1}^{3} q_{i k} y_{k}=0 \quad(i=1,2,3),
$$

where $p_{i k}$ and $q_{i k}$ are functions of the independent variable $x$, is left invariant in form by the transformations $\ddagger$

$$
\begin{aligned}
x & =\xi(x), \\
y_{i} & =\sum_{k=1}^{3} \alpha_{i k} \bar{y}_{k} \quad(i=1,2,3),
\end{aligned}
$$

where $\xi$ and $\alpha_{i k}$ are arbitrary functions of $x$, and where the determinant $\left|\alpha_{i k}\right|$ of the transformation (3) is different from zero. Moreover, these are the most general transformations which leave (1) invariant in form.

Let $\left(y_{1 i}, y_{2 i}, y_{3 i}\right)(i=1,2, \cdots, 6)$ be a fundamental system of solutions of equations (1). If the six functions $y_{1 i}(i=1,2, \cdots, 6)$ of $x$ are interpreted as the homogeneous coördinates of a point $\S y_{1}$ in 5 -space, this point generates a curve $C_{1}$ as $x$ varies. Likewise, the points $y_{2}$ and $y_{3}$ generate two curves $C_{2}$ and $C_{3}$, respectively. Corresponding points on the three curves, that is, points for which $x$ has the same value, determine a plane and as $x$ varies this plane generates a three spread $S_{3}$ of which $C_{1}, C_{2}, C_{3}$ are directrix curves. Since $\left(y_{1 i}, y_{2 i}, y_{3 i}\right)$ form a fundamental system of solutions of (1) it is impossible to find six functions $\lambda_{j}, \mu_{j}(j=1,2,3)$ such that the equations

$$
\sum_{j=1}^{3} \lambda_{j} y_{j i}+\sum_{j=1}^{3} \mu_{j} y_{j i}^{\prime}=0 \quad(i=1,2, \cdots, 6)
$$

* A. Ranum, On the projective differential geometry of $n$-dimensional spreadsgeneratedby $\infty^{1}{ }^{1}$ fats, Annali di Matematica Pura ed Applicata, (3), vol. 19 (1912), pp. 205-249; On the projective differential classification of $n$-dimensional spreads generated by $\infty^{1}$ flats, American Journal of Mathematics, vol. 37 (1915), pp. 117-158; On the differential geometry of ruled surfaces in 4-space and cyclic surfaces in 3-space, these Transactions, vol. 16 (1915, pp. 89-110.)

$\dagger$ E. Bompiani, Alcune proprietd proiettivo-diffenziali dei sistemi di rette negli iperspazi, Rendiconti del Circolo Matematico di Palermo, vol. 37 (1914), pp. 305-331.

$\ddagger$ Cf. Wilczynski, Projective Differential Geometry of Curves and Ruled Surfaces, Leipzig, 1906.

$\S$ Where no confusion can arise a point whose coördinates are of the form $\alpha_{i}(i=1,2, \cdots, 6)$ will be denoted simply by $\alpha$. 
are verified. This is equivalent to saying that $S_{3}$ is regular, that is, that consecutive generating planes of $S_{3}$ do not in general have a point in common.

The fact that all fundamental systems of solutions of (1) are linearly related shows that the three spreads obtained from different fundamental systems are all projectively related.

If we now consider the line determined by corresponding points of two of the curves, say $C_{1}$ and $C_{2}$, there is generated, as $x$ varies, a ruled surface $S$ which is on $S_{3}$ and of which $C_{1}$ and $C_{2}$ are directrix curves. Since consecutive generating planes of $S_{3}$ cannot have a point in common, consecutive generators of $S$ cannot have a point in common. In other words, $S$ cannot be developable in the ordinary sense.

Any non-developable ruled surface in 5-space may be considered as being on a regular three spread. Any two directrix curves of the ruled surface may be selected as directrix curves of a three spread $S_{3}$ and any other curve in 5-space may be chosen as the third directrix curve of $S_{3}$, provided merely that the tangents to the three curves at corresponding points do not lie in a 4-space. This selection is always possible since three lines in 5 -space do not in general lie in a 4 -space.

Suppose now that we have given a non-developable ruled surface $S$ which is determined by two directrix curves $C_{1}$ and $C_{2}$ of a three spread $S_{3}$ defined by the system of equations (1). The transformation (2) merely changes the parametric representation of the directrix curves and has no effect either on $S_{3}$ or $S$; but the transformation (3) shifts the directrix curves on $S_{3}$ from $C_{1}, C_{2}, C_{3}$ into three new curves $\bar{C}_{1}, \bar{C}_{2}, \bar{C}_{3}$ in such a way that $\bar{C}_{1}$ and $\bar{C}_{2}$ are on $S$ if and only if $\alpha_{13}=\alpha_{23}=0$. Thus the most general transformation (3) which leaves $S$ unchanged is given by the equations

$$
\begin{aligned}
& y_{1}=\alpha_{11} \bar{y}_{1}+\alpha_{12} \bar{y}_{2}, \\
& y_{2}=\alpha_{21} \bar{y}_{1}+\alpha_{22} \bar{y}_{2}, \\
& y_{3}=\alpha_{31} \bar{y}_{1}+\alpha_{32} \bar{y}_{2}+\alpha_{33} \bar{y}_{3}, \quad \alpha_{33}\left|\begin{array}{ll}
\alpha_{11} & \alpha_{12} \\
\alpha_{21} & \alpha_{22}
\end{array}\right| \neq 0 .
\end{aligned}
$$

Inasmuch as $S$ is independent of the nature of $C_{3}$, there is no loss of generality in putting $\alpha_{31}=\alpha_{32}=0$, and in selecting for $C_{3}$ a straight line, so that the last equation of (1) takes the simple form

$$
y_{3}{ }^{\prime \prime}+2 p_{33} y_{3}{ }^{\prime}+q_{33} y_{3}=0 .
$$


It is possible to study the projective differential properties of any nondevelopable rulea surface in 5-space by means of the system of equations

$$
\begin{aligned}
& y_{1}^{\prime \prime}+2 p_{11} y_{1}{ }^{\prime}+2 p_{12} y_{2}{ }^{\prime}+2 p_{13} y_{3}{ }^{\prime}+q_{11} y_{1}+q_{12} y_{2}+q_{13} y_{3}=0, \\
& y_{2}{ }^{\prime \prime}+2 p_{21} y_{1}{ }^{\prime}+2 p_{22} y_{2}{ }^{\prime}+2 p_{23} y_{3}{ }^{\prime}+q_{21} y_{1}+q_{22} y_{2}+q_{23} y_{3}=0, \\
& y_{3}{ }^{\prime \prime}+2 p_{33} y_{3}{ }^{\prime}+q_{33} y_{3}=0,
\end{aligned}
$$

under the transformations (2) and

(6)

$$
\begin{aligned}
& y_{1}=\alpha_{11} \bar{y}_{1}+\alpha_{12} \bar{y}_{2}, \\
& y_{2}=\alpha_{21} \bar{y}_{1}+\alpha_{22} \bar{y}_{2}, \\
& y_{3}=\quad \alpha_{83} \bar{y}_{3},
\end{aligned}
$$

We shall frequently have occasion to make use of the result of transforming (5) by (6). If the new coefficients are denoted by $\bar{p}_{i j}$ and $\bar{q}_{i j}$, we find by direct substitution that

$$
\begin{aligned}
& \Delta \bar{p}_{i j}=\sum_{k=1}^{2} A_{k i}\left[\alpha_{k j}^{\prime}+\sum_{l=1}^{2} p_{k l} \alpha_{l i}\right] \text {, } \\
& \Delta \bar{q}_{i j}=\sum_{k=1}^{2} A_{k i}\left[\alpha_{k i}^{\prime \prime}+\sum_{l=1}^{2}\left(2 p_{k l} \alpha_{l i}^{\prime}+q_{k l} \alpha_{l i}\right)\right] \text {, } \\
& \Delta \bar{p}_{i s}=\alpha_{38}: \sum_{k=1}^{2} A_{k i} p_{k s} \text {, } \\
& \Delta \bar{q}_{i 3}=\alpha_{33}^{\prime} \sum_{k=1}^{2} 2 A_{k i} p_{k 3}+\alpha_{33} \sum_{k=1}^{2} A_{k i} q_{k 3} \quad(i, j=1,2), \\
& \alpha_{38} \bar{p}_{38}=\alpha_{33}^{\prime}+\alpha_{33} p_{33} \\
& \alpha_{33} \bar{q}_{38}=\alpha_{33}^{\prime \prime}+2 \alpha_{33}^{\prime} p_{33}+\alpha_{33} q_{33},
\end{aligned}
$$

where $A_{i j}$ denotes the algebraic minor of $\alpha_{i j}$ in the determinant

$$
\Delta=\left|\begin{array}{ll}
\alpha_{11} & \alpha_{12} \\
\alpha_{21} & \alpha_{22}
\end{array}\right| .
$$

Again, the transformation (2) changes $p_{i j}$ and $q_{i j}$ into $\bar{p}_{i j}$ and $\bar{q}_{i j}$, where

$$
\begin{array}{rlr}
\bar{p}_{i i} & =\frac{1}{\xi^{\prime}}\left(p_{i i}+\frac{1}{2} \eta\right), \\
\bar{p}_{i j} & =\frac{1}{\xi^{\prime}} p_{i i} & (i \neq j), \\
\bar{q}_{i j} & =\frac{1}{\left(\xi^{\prime}\right)^{2}} q_{i j} & (i, j=1,2,3),
\end{array}
$$


with

$$
\eta=\frac{\xi^{\prime \prime}}{\xi^{\prime}} .
$$

The surface $S$ is singular if and only if each set of three consecutive generators lies in a 4 -space, that is, if and only if there exists a linear relation between $y_{1}, y_{2}, y_{1}^{\prime}, y_{2}^{\prime}, y_{1}^{\prime \prime}, y_{2}^{\prime \prime}$. The first two equations of (5) show that such a relation exists if and only if

$$
p_{13} q_{23}-p_{23} q_{13}=0 \text {. }
$$

From (7) and (8) it is evident that (9) is an invariant relation. Moreover, we see from (7) that it is always possible if (9) is satisfied, to transform (5) in such a way that in the new system either $p_{13}=q_{13}=0$ or $p_{23}=q_{23}=0$. We shall in our future study of singular ruled surfaces assume that the equations (5) are of the simple form*

$$
\begin{aligned}
& y_{1}^{\prime \prime}+2 p_{11} y_{1}^{\prime}+2 p_{12} y_{2}^{\prime} \quad+q_{11} y_{1}+q_{12} y_{2}=0, \\
& y_{2}^{\prime \prime}+2 p_{21} y_{1}^{\prime}+2 p_{22} y_{2}^{\prime}+2 p_{23} y_{3}^{\prime}+q_{21} y_{1}+q_{22} y_{2}+q_{23} y_{3}=0, \\
& y_{3}^{\prime \prime}+2 p_{33} y_{3}{ }^{\prime} \quad+q_{33} y_{3}=0 \text {. }
\end{aligned}
$$

It is possible for the invariant conditions $p_{13}=q_{13}=p_{23}=q_{23}=0$ to be satisfied. In this case the first two equations of (5) or (10) show that the surface lies entirely in a 3-space. The resulting system has already been used by Wilczynski for the study of such ruled surfaces. Consequently, we shall assume that in equations (10) $p_{23}$ and $q_{23}$ do not both vanish identically.

A reference to equations ( 7 ) shows that the conditions $p_{13}=q_{13}=0$ are left undisturbed by the transformation (6) of the dependent variables if and only if $\alpha_{12}=0$. Therefore in our study of singular surfaces we shall limit this transformation to the form

$$
\begin{aligned}
& y_{1}=\alpha_{11} \bar{y}_{1}, \\
& y_{2}=\alpha_{21} \bar{y}_{1}+\alpha_{22} \bar{y}_{2}, \\
& y_{3}=\quad \alpha_{33} \bar{y}_{3} .
\end{aligned}
$$

The conditions $p_{13}=q_{13}=0$ are undisturbed by the most general transformation (2) of the independent variable.

* The variable $y_{3}$ and its derivatives can of course be eliminated from (10). The form (10) has, however, the advantage in symmetry and in the fact that the same system may be used even if the surface lies wholly in a 4 -space by simply imposing an invariant condition on the coefficients. 


\section{The transversal SURFACE AND ASSOCIATED CURVES}

The analytical conditions $p_{13}=q_{13}=0$ which have been imposed in order to obtain (10) evidently determine for $C_{1}$ a unique curve on the singular surface $S$. We shall denote it by $C$. The first equation of (10) shows that the osculating plane of $C$ at a point $P$ lies in the 3 -space determined by the generator of $S$ through $P$ and the next consecutive generator of $S$. In other words, $C$ is the unique curve at each point of which there exists a tangent to $S$ intersecting three consecutive generators of $S$. It is evident geometrically that there can be only one such curve $C$ unless the surface lies entirely in a 3-space, in which case it is true for all curves on the surface.

Let $P$ be the point of intersection of $C$ and the generator corresponding to $x=x_{0}$ and let $y_{1}+\alpha(x) y_{2}$ be the curve on the surface at $P$ to which the line intersecting three consecutive generators is tangent. A point on the plane osculating this curve at $P$ is given by $y_{1}^{\prime \prime}+\alpha y_{2}^{\prime \prime}+2 \alpha^{\prime} y_{2}{ }^{\prime}+\alpha^{\prime \prime} y_{2}$, which point is seen by (10) to be on the tangent plane to $S$ at $P$ if and only if $\alpha\left(x_{0}\right)=0$ and $\alpha^{\prime}\left(x_{0}\right)=p_{12}\left(x_{0}\right)$. Thus a point on the tangent intersecting three consecutive generators is given by

$$
\tau=y_{1}{ }^{\prime}+p_{12} y_{2} .
$$

As $x$ varies the line determined by the points $y_{1}$ and $\tau$ generates a ruled surface $T$, the transversal surface* of $S$. The curve $C$ is the intersection of $S$ and $T$.

If the invariant condition $p_{12}=0$ is satisfied, $\tau=y_{1}^{\prime}$. We thus have the following theorem:

The identical vanishing of the invariant $p_{12}$ is the necessary and sufficient condition that a singular ruled surface $S$ in 5-space possess a curve which is asymptotic in the ordinary sense and that the transversal surface associated with $S$ be developable.

It is easily verified directly that $u_{12}=p_{12}^{\prime}-q_{12}+p_{11} p_{12}+p_{12} p_{22}$ is an invariant. Since $p_{12}=u_{12}=0$ is equivalent to $p_{12}=q_{12}=0$ there follows immediately from equation (10) the additional theorem

A singular ruled surface in 5-space possesses a straight line directrix if and only if the invariants $p_{12}$ and $u_{12}$ vanish simultaneously.

Ranum $\dagger$ uses an equation of nearly the same form as the first equation of (10) for the classification of singular ruled surfaces in 4-space and 5-space.

* Cf. Bompiani, loc. cit., p. 308.

$\dagger$ Cf. Ranum, American Journal of Mathematics, vol. 37 (1915), pp. 139-146 and 150-151. 
The classification depends upon the vanishing of the coefficients corresponding to $p_{12}$ and $q_{12}$.

The surface $T$, when not a developable, has associated with it a system of equations of the form of (1) in terms of the variables $y_{1}, \tau, y_{3}$. Assuming, accordingly, that $p_{12} \neq 0$, we may obtain one of its equations by eliminating $y_{2}$ and $y_{2}^{\prime}$ from the first equation of (10) by means of (12). It is

$$
y_{1}^{\prime \prime}-\frac{2}{p_{12}}\left(p_{12}^{\prime}-\frac{1}{2} q_{12}+p_{11} p_{12}\right) y_{1}^{\prime}-2 \tau^{\prime}-q_{11} y_{1}+\frac{1}{p_{12}}\left(2 p_{12}^{\prime}-q_{12}\right) \tau=0 .
$$

This equation shows at once that $T$ is also singular and that $C$ is its curve of intersection with its transversal surface. Moreover, since the expression for $T$ corresponding to $\tau$ for $S$ is

$$
y_{1}^{\prime}-\tau=-p_{12} y_{2},
$$

the transversal surface of $T$ must be $S$. Thus we have a theorem* which holds for all singular ruled surfaces in 5-space, including all ruled surfaces in 4-space, whose transversal surfaces are not developables:

The relation between a singular ruled surface and its transversal surface is reciprocal.

Let us designate by $d=y_{1}^{\prime}+\alpha y_{1}+\beta y_{2}$ a point on the tangent plane to $S$ at $y_{1}$. As $x$ varies, $d$ will generate a curve $D$. On differentiating twice we find that the plane osculating $D$ at $d$ is the tangent plane to $S$ at $y$ if

$$
\alpha=\frac{1}{2 p_{12}}\left(2 p_{12}^{\prime}-q_{12}+4 p_{11} p_{12}\right), \quad \beta=2 p_{12} .
$$

Consequently, the tangent planes osculate the curve $D$ given by

$$
d=y_{1}^{\prime}+\frac{1}{2 p_{12}}\left(2 p_{12}^{\prime}-q_{12}+4 p_{11} p_{12}\right) y_{1}+2 p_{12} y_{2}
$$

or

$$
d=\tau+\frac{1}{2 p_{12}}\left(2 p_{12}^{\prime}-q_{12}+4 p_{11} p_{12}\right) y_{1}+p_{12} y_{2} .
$$

The line joining $y_{1}$ and $d$ intersects the line determined by $\tau$ and $y_{2}$ at $\tau+p_{12} y_{2}$. Since $y_{1}^{\prime}=\tau-p_{12} y_{2}$, we have the following theorem: $\dagger$

* This theorem is well known for surfaces in 4-space. Cf. Bompiani, loc. cit.

$\dagger$ Ranum has proved this theorem for surfaces in 4-space. Cf. these Transactions, vol. 16 (1915), p. 92. 
The line determined by $y_{1}$ and $d$ is the harmonic conjugate of the tangent to $C$ at $y_{1}$ with respect to the generator of $S$ through $y_{1}$ and the corresponding generator of $T$.

It is easy to obtain an infinite set of pairs of surfaces and transversal surfaces which have the same curves $C$ and $D$ and for which the above theorem holds. In order to show this fact let us put

$$
e=d+k y_{1}^{\prime}, \quad f=d-k y_{1}^{\prime},
$$

where $k$ is a constant. It is evident that the lines $y_{1} e$ and $y_{1} f$ are harmonic with respect to the lines $y_{1} d$ and $y_{1} y_{1}^{\prime}$. The ruled surface generated by $y_{1} e$ has for one of its equations

$$
\begin{gathered}
2 p_{12} k y_{1}^{\prime \prime}-k\left(2 p_{12}^{\prime}-q_{12}\right) y_{1}^{\prime}-2 p_{12} e^{\prime}+\left[2 p_{12} \alpha^{\prime}-2 p_{12} q_{11}-\alpha\left(2 p_{12}^{\prime}-q_{12}\right)\right] y_{1} \\
+\left(2 p_{12}^{\prime}-q_{12}\right) e=0,
\end{gathered}
$$

where

$$
\alpha=\frac{1}{2 p_{12}}\left(2 p_{12}^{\prime}-q_{12}+4 p_{11} p_{12}\right) .
$$

Thus, it is seen to be a singular surface which intersects its transversal surface in $C$. Since a point on the generator of its transversal surface is given by $-f / 2 k$ and its point corresponding to $d$ for $S$ by $-d / k$, our statement is proved. This condition is the exact analogy of the relation between an infinite set of ruled surfaces in 3-space which have the same flecnode curve.* $^{*}$

\section{SURFACES IN 4-SPACE}

Our ruled surface $S$ lies entirely in a 4 -space if every set of four consecutive generators is contained in a 4-space. Consequently, it must be possible in this case to express $y_{1}^{(3)}$ and $y_{2}^{(3)}$ in terms of $y_{1}^{\prime \prime}, y_{2}^{\prime \prime}, y_{1}^{\prime}, y_{2}^{\prime}, y_{1}, y_{2}$. It is always possible thus to express $y_{1}^{(3)}$ but $y_{3}^{\prime \prime}, y_{3}^{\prime}, y_{3}$ can be eliminated from the equation resulting from the differentiation of the second equation of (10) only if

$$
\varphi_{4}=2\left(p_{23}^{\prime} q_{23}-p_{23} q_{23}^{\prime}\right)+4 p_{23}\left(p_{23} q_{33}-q_{23} p_{33}\right)+q_{23}^{2}=0 .
$$

It is evident from its geometrical significance that the expression (16) is a relative invariant, a fact which may also be verified directly by means of equations (7) and (8).

If $S$ lies in a 4 -space but not in a 3 -space, $p_{23} \neq 0$, a fact which is evident from (16) when it is remembered that $p_{23}=q_{23}=0$ are the conditions that $S$

* Cf. Wilczynski, loc. cit., p. 233. 
lie entirely in a 3 -space. In fact, if $S$ is not in a 3 -space, it is always possible to select $C_{3}$ in such a way that $p_{23} \neq 0$ regardless of whether $S$ is in a 4 -space or 5-space. The second equation of (10) shows that it is only necessary to choose the point on $C_{3}$ corresponding to $y_{1}$ and $y_{2}$ on $C$ and $C_{2}$ in such a way that it is not in the 4 -space determined by the tangent to $C$ at $y_{1}$ and the osculating plane to $C_{2}$ at $y_{2}$.

The transformation of $q_{23}$ as given by equations (7) shows that we may obtain a set of equations in which $q_{23}$ vanishes by selecting $\alpha_{33}$ to satisfy the equation $2 \alpha_{33}^{\prime} p_{23}+\alpha_{33} q_{23}=0$. This choice of $\alpha_{33}$ is always possible when $p_{23} \neq 0$. Moreover, the relation $q_{23}=0$ is maintained if $\alpha_{33}=$ const. When $p_{23} \neq 0$ and $q_{23}=0$ the relation (16) reduces to $q_{33}=0$. We thus have the following theorem:

The necessary and sufficient condition that $S$ lie entirely in 4-space is $\varphi_{4}=0$. If $S$ does not lie in a 3-space, this invariant relation may be always reduced to $q_{33}=0$ by the transformation of (10) into a system for which $q_{23}=0$.

Equations (10) with $q_{23}=q_{33}=0$ may be used to show analytically that there are $\infty^{2}$ curves on a ruled surface in 4-space whose osculating 3-space at a given point contains the tangent plane to $S$ at the point.* Differentiation of the second equation of (10) shows that with $q_{23}=q_{33}=0$ we can express $y_{1}$ in terms of $y_{2}^{(3)}, y_{2}^{\prime \prime}, y_{2}^{\prime}, y_{2}$ if and only if

$$
M=p_{23}\left(2 p_{21}^{\prime}+q_{21}-4 p_{11} p_{21}\right)-p_{21}\left(2 p_{23}^{\prime}-4 p_{23} p_{33}\right)=0 .
$$

If in the transformation (11) we put $\alpha_{11}=\alpha_{22}=\alpha_{33}=1$, equations (7) show that $\alpha_{21}$ must satisfy a differential equation of the second order, in order that the transformed form of $M$ shall equal zero. Our statement above is thus proved.

\section{The CURVE OF INTERSECtion OF $S$ AND $T$}

The differential equation for $C$ is of the sixth order in $y_{1}$. By taking successive derivatives of the first equation of (10) and eliminating $y_{2}$, $y_{3}$ and their derivatives we have for the desired equation

$$
\left|\begin{array}{ccccc}
y_{1}{ }^{(6)}+l_{16} y_{1}^{\prime}+m_{16} y_{1} & l_{26} & l_{36} & m_{26} & m_{36} \\
y_{1}{ }^{(5)}+l_{15} y_{1}^{\prime}+m_{15} y_{1} & l_{25} & l_{35} & m_{25} & m_{35} \\
y_{1}{ }^{(4)}+l_{14} y_{1}^{\prime}+m_{14} y_{1} & l_{24} & l_{34} & m_{24} & m_{34} \\
y_{1}{ }^{(3)}+l_{13} y_{1}^{\prime}+m_{13} y_{1} & l_{23} & l_{33} & m_{23} & m_{33} \\
y_{1}{ }^{\prime \prime}+2 p_{11} y_{1}^{\prime}+q_{11} y_{1} & 2 p_{12} & 0 & q_{12} & 0
\end{array}\right|=0,
$$

* Cf. Bompiani, loc. cit., pp. 310-311. 
where

with

$$
\begin{array}{ll}
l_{i j} & =l_{i, j-1}^{\prime}+m_{i, j-1}-2 \sum_{k=1}^{3} p_{k i} l_{k, j-1}, \\
m_{i j} & =m_{i, j-1}^{\prime}-\sum_{k=1}^{3} q_{k i} l_{k, j-1} \quad(j=3,4,5,6),
\end{array}
$$

$$
l_{i 2}=2 p_{1 i}, \quad m_{i 2}=q_{1 i} .
$$

Equation (17) may be used for the study of the various projective differential properties of the curve $C$. In particular, we shall use it for the determination of the conditions under which $C$ is located in the various sub-spaces.

(a) $C$ a straight line. It has already been seen that the vanishing of the invariants $p_{12}$ and $u_{12}$ is the necessary and sufficient condition. This fact is also evident directly from (17).

(b) $C$ in a 2-space but not a straight line. Since $p_{12}$ and $q_{12}$ cannot both vanish identically, $l_{33}=m_{33}=l_{23} q_{12}-2 p_{12} m_{23}=0$. It follows at once from (18) that $p_{23}=q_{23}=0$. Therefore, $S$ must be in a 3 -space and the invariant

$$
\Delta_{2}=l_{23} q_{12}-2 p_{12} m_{23}=2\left(p_{12}^{\prime} q_{12}-p_{12} q_{12}^{\prime}\right)+4 p_{12}\left(p_{12} q_{22}-p_{22} q_{12}\right)+q_{12}^{2}
$$

must vanish identically. The fact that $\Delta_{2}$ is an invariant is evident geometrically and may be verified directly.

(c) $C$ in a 3-space but not in a 2-space. The fact that $p_{12}$ and $q_{12}$ cannot both vanish shows that

$$
l_{34} m_{33}-l_{33} m_{34}=4 \underset{p_{12} \varphi_{4}}{2}=0 .
$$

Thus, either $\varphi_{4}=0$ and $S$ is in a 4 -space, or $p_{12}=0$ and $T$ is a developable. In the latter case the two additional equations which must be satisfied reduce immediately to $2 p_{23} q_{12}^{3}=0$ and $q_{23} q_{12}^{3}=0$, and the surface $S$ is therefore in a 3 -space.

If $\varphi_{4}=0$ and $p_{12} \neq 0$, we must have also

$$
\Delta_{3}=\left|\begin{array}{ccc}
l_{24} & l_{34} & m_{24} \\
l_{23} & l_{33} & m_{23} \\
2 p_{12} & 0 & q_{12}
\end{array}\right|=0, \quad \Delta_{3}^{\prime}=\left|\begin{array}{ccc}
l_{24} & m_{24} & m_{34} \\
l_{23} & m_{23} & m_{33} \\
2 p_{12} & q_{12} & 0
\end{array}\right|=0 .
$$

But

$$
2 p_{23} \Delta_{3}{ }^{\prime}=-q_{23} \Delta_{3}+2 p_{12} \varphi_{4}\left(l_{23} q_{12}-2 m_{23} p_{12}\right)
$$

and therefore $\Delta_{3}^{\prime}$ vanishes as a consequence of the vanishing of $\Delta_{3}$ and $\varphi_{4}$. It is easily verified by direct substitutions that $\Delta_{3}$ is an invariant. 
(d) C in a 4-space but not in a 3-space. It is necessary and sufficient that the determinant

$$
\left|\begin{array}{cccc}
l_{25} & l_{35} & m_{25} & m_{35} \\
l_{24} & l_{34} & m_{24} & m_{34} \\
l_{23} & l_{33} & m_{23} & m_{33} \\
2 p_{12} & 0 & q_{12} & 0
\end{array}\right|
$$

shall vanish. It is geometrically evident that the determinant must be an invariant. If the transversal surface is developable, $p_{12}=0$ and the above determinant reduces to $q_{12}^{2} \varphi_{4}$. It follows that in this case the surface $S$ lies in a 4-space.

\section{SEMI-CANONICAL FORMS OF THE EQUATIONS}

Equations (7) show that the transformation (11) converts the coefficients $p_{11}, p_{21}, p_{22}, p_{33}$ into $\bar{p}_{11}, \bar{p}_{21}, \bar{p}_{22}, \bar{p}_{33}$ where

$$
\begin{aligned}
\Delta \bar{p}_{11} & =\alpha_{22}\left(\alpha_{11}^{\prime}+\alpha_{11} p_{11}+\alpha_{21} p_{12}\right) \\
\Delta \bar{p}_{21} & =\alpha_{11}\left(\alpha_{21}^{\prime}+\alpha_{11} p_{21}+\alpha_{21} p_{22}\right)-\alpha_{21}\left(\alpha_{11}^{\prime}+\alpha_{11} p_{11}+\alpha_{21} p_{12}\right) \\
\Delta \bar{p}_{22} & =\alpha_{11} \alpha_{22}^{\prime}+\alpha_{11} \alpha_{22} p_{22}-\alpha_{21} \alpha_{22} p_{12} \\
\alpha_{33} \bar{p}_{33} & =\alpha_{33}^{\prime}+\alpha_{33} p_{33}
\end{aligned}
$$

If $\alpha_{11}$ and $\alpha_{21}$ are selected as a pair of solutions of the equations

$$
\begin{aligned}
& \alpha_{11}^{\prime}+\alpha_{11} p_{11}+\alpha_{21} p_{12}=0 \\
& \alpha_{21}^{\prime}+\alpha_{11} p_{21}+\alpha_{21} p_{22}=0,
\end{aligned}
$$

the transformed coefficients $\bar{p}_{11}$ and $\bar{p}_{21}$ become zero. Furthermore, if the value of $\alpha_{11}$ and $\alpha_{21}$ thus determined are substituted in the equation

$$
\alpha_{11} \alpha_{22}^{\prime}+\alpha_{11} \alpha_{22} p_{22}-\alpha_{21} \alpha_{22} p_{12}=0
$$

and $\alpha_{22}$ is selected as a solution of the resulting equation, the coefficient $\bar{p}_{22}$ also becomes zero. Finally, $\bar{p}_{33}$ becomes zero if $\alpha_{33}$ is selected to satisfy the equation $\alpha_{33}^{\prime}+\alpha_{33} p_{33}=0$.

Equations (10) may thus always be transformed into the simpler form

$$
\begin{aligned}
& y_{1}^{\prime \prime}+2 p_{12} y_{2}^{\prime}+q_{11} y_{1}+q_{12} y_{2}=0, \\
& y_{2}^{\prime \prime}+2 p_{23} y_{3}^{\prime}+q_{21} y_{1}+q_{22} y_{2}+q_{23} y_{3}=0, \\
& y_{3}^{\prime \prime}+q_{33} y_{3}=0 .
\end{aligned}
$$


In accordance with the terminology of Wilczynski, we shall call (20) the semi-canonical form of equations (10).

We have already seen that it is always possible to select $\alpha_{33}$ in such a way as to make $q_{23}$ vanish if $p_{23} \neq 0$. Consequently, we have a second semicanonical form in which $q_{23}$ vanishes in place of $p_{33}$.

\section{INVARIANTS AND COVARIANTS}

Clearly every invariant and covariant of the general system (1) under the transformations (2) and (3) either vanishes identically or is an invariant or covariant of the special system (10) under the transformations (2) and (11). Moreover, a comparison of the form of equations (7) and (8) with the corresponding transformations of the coefficients of the system similar to (1), but with $i=1,2$, shows that every invariant and covariant of the latter system either vanishes identically, or represents also an invariant or covariant of (10). Complete systems of invariants and covariants have been calculated* for the system (1) for any value of $i$ under the most general transformations which leave such a system invariant in form.

Making use of these facts and of equations (7) and (8), and remembering the invariants already discovered, we see easily that the following expressions are relative invariants:

$$
\begin{aligned}
& p_{12}, u_{12}, \quad p_{23}, \\
& \theta_{2}=\left(u_{11}-u_{22}\right)^{2}+4 u_{12} u_{21}, \\
& \vartheta_{5}=8 \theta_{2}^{\prime \prime} \theta_{2}-9\left(\theta_{2}^{\prime}\right)^{2}+32\left(u_{11}+u_{22}\right) \theta_{2}^{2}, \\
& \varphi_{4}=2\left(p_{23}^{\prime} q_{23}-p_{23} q_{23}^{\prime}\right)+4 p_{23}\left(p_{23} q_{33}-p_{33} q_{23}\right)+q_{23}^{2}, \\
& \Delta_{2}=l_{23} q_{12}-2 p_{12} m_{23}=2\left(p_{12}^{\prime} q_{12}-q_{12}^{\prime} p_{12}\right)+4 p_{12}\left(p_{12} q_{22}-q_{12} p_{22}\right)+q_{12}^{2}, \\
& \Delta_{3}=\left|\begin{array}{ccc}
l_{24} & l_{34} & m_{24} \\
l_{23} & l_{33} & m_{23} \\
2 p_{12} & 0 & q_{12}
\end{array}\right|,
\end{aligned}
$$

where

$$
u_{i k}=p_{i k}^{\prime}-q_{i k}+\sum_{j=1}^{2} p_{i j} p_{j k} \quad(i, k=1,2),
$$

and where $l_{i k}$ and $m_{i k}$ are defined by equations (18).

It is easy to verify that the above invariants are a complete system provided merely that certain of them are not zero. In order to do this let us

* Cf. Invariants and covariants. 
assume that (10) has been transformed into a semi-canonical form so that $p_{11}=p_{22}=p_{21}=0$ and either $p_{33}=0$ or $q_{23}=0$. Then if the invariants (21) are known functions of $x$, with $p_{12}, p_{23}, \theta_{2}, u_{12}, \Delta_{2}$ not equal to zero, it is possible to solve for the coefficients of the semi-canonical form. The coefficients $p_{12}, p_{23}, q_{12}=p_{12}-u_{12}$ are known at once. The expression for $q_{22}$ follows immediately from $\Delta_{2}$. Since, in either semi-canonical form $u_{11}+u_{22}=$ $-q_{11}-q_{22}$ and $u_{21}=-q_{21}$, the expression for $q_{11}$ can be obtained from $\vartheta_{5}$ and then the expression for $q_{21}$ from $\theta_{2}$. Since $q_{33}$ does not appear in $\Delta_{3}$ and since $p_{33}, q_{23}$ occur only in $l_{34}$ which, with $p_{11}=p_{22}=p_{21}=0$, reduces to

$$
-2 p_{23}\left(2 p_{12}^{\prime}+q_{12}\right)+8 p_{12} p_{23} p_{33}-4 p_{12} p_{23}^{\prime}-4 p_{12}^{\prime} p_{23}-2 p_{12} q_{23},
$$

it is possible to obtain the expression for that one of the two coefficients $p_{33}$ and $q_{23}$ which has not been made zero. Finally, $\varphi_{4}$ gives the expression for $q_{33}$.

Since higher derivatives of $y_{i}$ than the second can be eliminated by means of equations (10) there can be only six independent covariants in addition to the complete system of invariants. Two of these are evidently $y_{1}$ and $y_{3}$. Three others may be obtained immediately from the complete set for the system of equations of form (1) with two independent variables:*

$$
\begin{aligned}
R_{2} & =\left|\begin{array}{cc}
y_{1} & y_{2} \\
r_{12} & r_{22}
\end{array}\right|, \quad T_{12}=\left|\begin{array}{cc}
y_{1} & y_{2} \\
t_{12} & t_{22}
\end{array}\right|, \\
\psi_{0} & =4 T_{y 2}+2\left(u_{11}+u_{22}\right) T_{12}+S_{12},
\end{aligned}
$$

where

$$
T_{y 2}=\left|\begin{array}{cc}
t_{12} & t_{22} \\
r_{12} & r_{22}
\end{array}\right|, \quad S_{12}=\left|\begin{array}{cc}
y_{1} & y_{2} \\
s_{12} & s_{22}
\end{array}\right|
$$

and

$$
r_{i 2}=\sum_{j=1}^{2} u_{i j} y_{j}, \quad t_{i 2}=y_{i}{ }^{\prime}+\sum_{j=1}^{2} p_{i j} y_{i}, \quad s_{i 2}=\sum_{j=1}^{2} v_{i j} y_{j} \quad(i=1,2),
$$

and

$$
\text { (26) } u_{i k}=p_{i k}^{\prime}-q_{i k}+\sum_{j=1}^{2} p_{i j} p_{j k}, v_{i k}=u_{i k}^{\prime}+\sum_{j=1}^{2}\left(p_{i j} u_{j k}-p_{j k} u_{i j}\right)(i, k=1,2) \text {. }
$$

* Cf. Invariants and covariants, pp. 346-352. 
The remaining covariant may be selected from the set for the system(1) with three independent variables:

where

$$
T_{23}=\left|\begin{array}{ccc}
y_{1} & y_{2} & y_{3} \\
r_{13} & r_{23} & r_{33} \\
t_{13} & t_{23} & t_{33}
\end{array}\right|
$$

$$
r_{i 3}=\sum_{j=1}^{3} u_{i j 3} y_{j}, \quad t_{13}=y_{i}{ }^{\prime}+\sum_{j=1}^{3} p_{i j} y_{i} \quad(i=1,2,3)
$$

and

$$
u_{i k 3}=p_{i k}^{\prime}-q_{i k}+\sum_{j=1}^{3} p_{i j} p_{j k} \quad(i, k=1,2,3) .
$$

The independence of the above set of covariants is easily shown by means of the functional determinant formed with respect to the variables $y_{i}, y_{i}^{\prime}(i=1,2,3)$.

\section{Derivative surfaces}

The expressions $t_{12}, t_{22}$ defined by (25) are cogredient with $y_{1}, y_{2}$ under the transformation (11). The line joining the points $t_{12}$ and $t_{22}$ generates a surface $S^{\prime}$ as $x$ varies and this surface we shall call the derivative surface of $S$. Since the transformation $\xi=\xi(x)$ of the independent variable converts $t_{12}$ and $t_{22}$ into $\bar{t}_{12}$ and $\bar{t}_{22}$, respectively, where

$$
\bar{t}_{12}=\frac{1}{\xi^{\prime}}\left(t_{12}+\frac{1}{2} \eta y_{1}\right), \quad \bar{t}_{22}=\frac{1}{\xi^{\prime}}\left(t_{22}+\frac{1}{2} \eta y_{2}\right), \quad \eta=\frac{\xi^{\prime \prime}}{\xi^{\prime}},
$$

the surface $S^{\prime}$ changes as $\eta$ changes. Since $t_{12}=\tau+p_{11} y_{1}$, the curve generated by $t_{12}$ is always on the transversal surface $T$.

We proceed to find the differential equations of form (5) for $S^{\prime}$. It is easily verified that

$$
\begin{aligned}
t_{12}^{\prime}+p_{11} t_{12}+p_{12} t_{22} & =u_{11} y_{1}+u_{12} y_{2}, \\
t_{22}^{\prime}+p_{21} t_{12}+p_{22} t_{22} & =u_{21} y_{1}+u_{22} y_{2}-2 p_{23} y_{3}{ }^{\prime}-q_{23} y_{3} .
\end{aligned}
$$

By differentiating each equation of (31) and eliminating $y_{1}$ and $y_{2}$ and their derivatives, we obtain a set of equations of form (5) in the variables $t_{12}$, $t_{22}, y_{3}$. If in these equations we denote the coefficients corresponding to the coefficients $p_{i j}$ and $q_{i j}$ of (5) by $P_{i j}$ and $Q_{i j}$, respectively, we find 


$$
\begin{aligned}
& I P_{11}=\frac{1}{2} p_{11} I-\frac{1}{2}\left(v_{11} u_{22}-v_{12} u_{21}-p_{11} I\right), \\
& I P_{12}=\frac{1}{2} p_{12} I+\frac{1}{2} A, \\
& I Q_{12}=p_{12}^{\prime} I-p_{12}\left(v_{11} u_{22}-v_{12} u_{21}-p_{11} I\right)-u_{12} I+p_{22} A, \\
& I P_{13}=p_{23} A, \quad I Q_{13}=q_{23} A, \\
& I P_{23}=p_{23} B+\frac{1}{2}\left(2 p_{23}^{\prime}+q_{23}-4 p_{23} p_{33}\right) I, \\
& I Q_{23}=q_{23} B+\left(q_{23}^{\prime}-2 p_{23} q_{33}\right) I,
\end{aligned}
$$

where

$$
\begin{aligned}
& I=u_{11} u_{22}-u_{12} u_{21} \\
& A=u_{12} v_{11}-u_{11} v_{12}+p_{12} I, \quad B=u_{12} v_{21}-u_{11} v_{22}+p_{22} I .
\end{aligned}
$$

Assuming* $I \neq 0$ we see at once that $S^{\prime}$ is singular if and only if

$$
P_{23} Q_{13}-P_{13} Q_{23}=\frac{A \varphi_{4}}{2 I}=0 .
$$

Thus $S^{\prime}$ is singular if $S$ is in a 4 -space, a fact geometrically evident, or if $A=0$. Equations (32) show that if $A=0$ the singular surface $S^{\prime}$ intersects its transversal in a curve on $T$. Now the transformations (11) leave $A$ unchanged in form but the transformation $\xi=\xi(x)$ converts $A$ into $\bar{A}$ where

$$
\bar{A}=\frac{1}{\left(\xi^{\prime}\right)^{5}}\left[A+\frac{1}{2} p_{12}\left(u_{11}+u_{22}\right) \mu-\frac{1}{2} v_{12} \mu+\frac{1}{4} p_{12} \mu^{2}+\frac{1}{2} u_{12} \mu^{\prime}\right]
$$

with

$$
\mu=\eta^{\prime}-\frac{1}{4} \eta^{2}
$$

We therefore have the following theorem:

If $S$ is not in a 4-space there are $\infty^{2}$ derivative surfaces which are singular and each of them intersects its transversal surface in a curve on $T$; if $S$ is in a 4-space there are $\infty^{2}$ of its singular derivative surfaces each of which intersects its transversal surface in a curve on $T$.

Let us assume that the independent variable has been so chosen that $A=0$. The point associated with $S^{\prime}$ which corresponds to $d$ for $S$ is given by

* Equations (31) show that $I \neq 0$ if $C_{3}$ is so selected as not to have a point in common with the tangent 3-space to $S^{\prime}$. 
(34)

$$
t_{12}^{\prime}+\frac{2 P_{12}^{\prime}-Q_{12}+4 P_{11} P_{12}}{2 P_{12}} t_{12}+2 P_{12} t_{22}=u_{11} y_{1}+u_{12} y_{2}+\frac{u_{12}}{p_{12}} t_{12}
$$

$$
=\frac{u_{12}}{p_{12}} d+\left(u_{11}-\frac{2 p_{12}^{\prime}-q_{12}+2 p_{11} p_{12}}{2 p_{12}} \cdot \frac{u_{12}}{p_{12}}\right) y_{1} .
$$

Thus the point is on the line of intersection of the tangent planes to $S$ at $y_{1}$ and to $S^{\prime}$ at $t_{12}$, and also on the line joining $y_{1}$ and $d$.

If the invariant $u_{12}$ vanishes but $C$ is not a straight line, that is, $p_{12} \neq 0$, we have $A=0$ only if $u_{11}=0$. It follows from equation (34) that in this case every singular derivative surface which intersects its transversal surface in a curve on $T$ is developable with its edge of regression on $T$.

Since $2 P_{12}=p_{12}$ when $A=0$, it follows that the transversal surface of a singular derivative surface which intersects its transversal surface in a curve on $T$ is developable if and only if $T$ is developable.

UNIVERSTTY OF KANSAS,

LaWRence, Kans. 